\title{
The Effect of Numbing Needles Pain with Topical Anaesthesia on Preterms' Exposure to Neonatal Sepsis
}

\author{
Rabab El-Sayed Hassan El-Sayed ${ }^{1}$, Samar El Hoseiny Abd Elraouf ${ }^{2}$

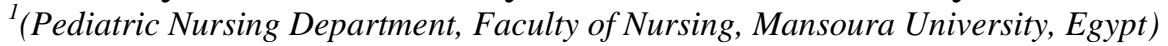 \\ ${ }^{2}$ (Community Health Nursing Department, Faculty of Nursing, Mansoura University, Egypt)
}

\begin{abstract}
In neonatal intensive care units, preterm infants are subjected to an average of two and up to fourteen painful procedures per day. Pain and infection are inextricably linked and always neonatal sepsis remains a feared cause of morbidity and mortality during the neonatal period. The study aimed to assess the effect of numbing needles pain with topical anaesthesia on preterms' exposure to neonatal sepsis. A quasiexperimental design was utilized. A purposive sample enrolled all premature infants $(n=72)$ who admitted to the neonatal care department of Mansoura University Children Hospital. Four tools were used for data collection, namely preterm infants' profile, follow-up and clinical outcome assessment sheets, and Premature Infant Pain Profile. The study findings illustrated that there was statistically significant relation between frequency of applying topical anaesthesia and immunologic parameters presented by average of daily weight gain, number of culture-proven infections and negative $C$-reactive protein weekly profile, at $p<.001$, and $p=.001$ respectively. As well there was statistically significant relation between premature infants' calm state during needle painful events and their $C$-reactive protein weekly profile, at $p<.001$. Topical anaesthesia is recommended for procedural pain management in the neonatal care units to reduce the adverse effect of pain on preterm infants' immunity.
\end{abstract}

Keywords: Needles Pain, NICU, Neonatal Sepsis, Preterm infants, Topical Anaesthesia

\section{Introduction}

Preterm infants, especially those born between $28-32$ weeks of gestation (very premature) are exposed to repeated procedural pain-related stress, during a period of physiological vulnerability and rapid brain development, as part of their life-saving care in the neonatal intensive care unit (NICU). [1] Carbajal et al. [2] reported that neonates underwent an average of 10 painful procedures daily and $91 \%$ of these procedures were performed without specific analgesia, despite cutaneous receptive fields are large in the neonate, and peripheral sensory fibers are sensitive to tissue injury. [3]

Assessment of neonatal pain is a pressing concern, especially within the context of neonatal intensive care where tiny infants may be exposed to prolonged and repeated pain during lengthy hospitalization. [4] For effective pain management, it is important to correctly identify the pain experienced by the newborns in time through the bedside nurse. Premature infant pain profile (PIPP) code a variety of behavioral and physiological responses (i.e. frown forehead, eye squeeze, naso-labial furrow, changes in heart rate, and oxygen saturation) in order to quantify pain in hospitalized neonates, who cannot self-reporting pain. [5 \& 6]

Nociception is the ability of peripheral afferent neurons to sense noxious stimuli. [7] Many studies reported that nociception and immune function exhibit a bidirectional relationship, in which each function affecting the other. [8-11] Two relevant literatures suggest applying effective analgesic strategies in the NICUs that may modulate immune function, and decrease the risk of neonatal infection. [12 \& 13] Moreover, Vinall and Grunau [14] presented recent work supporting that, greater exposure to painful procedures in the NICU has been associated with the reprogramming of the stress hormone and immune systems among premature infants. Therefore, alleviation of pain caused by invasive procedures in neonates is important for humane reasons as well as for avoiding the acute physiological instability (e.g., metabolic, immune).

Responses to neonatal pain vary based on gestational age, sleep-wake state, illness severity, as well as recency and duration of previous exposures to pain and non-invasive interventions. [15] Therefore, clinicians are faced with the difficult task of discriminating and appropriately managing pain in infants born premature. Every NICU should have a comprehensive approach to reduce pain for the admitted patients. Both nonpharmacological and pharmacological approaches play a role in the management of pain in the neonate, and while non-pharmacologic management is recommended as a first step, often invasive procedures in the NICU are still performed without support. [16] Unmanaged pain may have substantive effects on the developing brain, and stress systems of premature neonates; however, pain management remains a challenge.

The use of swaddling, sucrose, pacifiers, and decreased environmental stimuli have shown limited therapeutic efficacy in treating mild to moderate painful stimuli. Local and systemic analgesia are often the treatment of choice for procedural pain, and specifically the various topical anesthetic agents including 
Lidocaine infiltration, can be effective in managing bedside procedural pain without exposing the developing brain to the potential hazards of systemic anesthetics. [17]Topical local anesthetics are ideally suited for anesthetizing the skin in infants because most lack systemic side effects. Lidocaine spray is a topical local anesthetic preparation that offers several advantages over other preparations, including faster onset of action and lack of systemic adverse effects. [18 \& 19] It can effectively reduce pain from certain types of bedside procedural pain, such as a venipuncture, lumbar puncture, and intravenous catheter insertion in term and premature neonates, when applied for a sufficient length of time according to the medication associated usage instructions (onset time is 2-3 minutes) before the procedure. [20]

Significance of the study

Although the association between the central nervous system and immune function is well documented, how that relationship affects the incidence of infection is all but absent from the literature. Therefore, this study was aimed to: Assess the effect of numbing needles pain with topical anaesthesia on preterms' exposure to neonatal sepsis in the NICU.

\section{Research Hypotheses}

It is hypothesized that:

H1: Applying topical anaesthesia will reduce needle related pain intensity proven by low total PIPP scores of preterm infants.

H2: Topical numbing of needles pain will reduce the number of culture-proven infections among preterm infants throughout their stay in the NICU.

H3: Topical numbing of needles pain will show fewer behavioral signs of stress among preterm infants during needle related painful events.

H4: Topical numbing of needles pain will maintain physiologic stability of preterm infants immediately after needle related painful events.

The primary outcome of the study was to enhance the immune system of premature infants through report a reduction in the number of culture-proven infections during their stay in the NICU. Secondary outcomes included assessment of premature infants' behavior signs of stress; including change of their facial cues, physiologic stability, weight gain, and length of hospital stay.

\section{Subjects And Methods}

2.1 Design: A quasi-experimental design was used in this study.

2.2 Setting: Study subjects were recruited from the neonatal care department (NCD) of Mansoura University Children Hospital, Egypt. This department was internally classified into three separate units according to the severity of the admitted infants into: ordinary unit, intermediate care unit, and intensive care unit.

2.3 Subjects: A purposive sample enrolled all premature infants $(n=72)$ who admitted to the previously mentioned setting with their age between 26 to 36 weeks of gestation, throughout a period of 6 months; started from November 2015 to April 2016. Subjects were excluded if they had a history of necrotizing enterocolitis, receipt of steroids, history of congenital infection, contraindication to blood draw such as bleeding disorder or severe anemia, or receiving human milk feedings. Infants receiving human milk feedings were excluded because human milk is known to contain immune-related compounds that could potentially affect study outcome. [21] 2.4 Tools of data collection: The data will be collected by using four tools. First, second and third tools were designed by the researchers after reviewing the relevant literatures, while fourth tool was adopted from Ballantyne et al. and McNair et al. [22 \& 23], as the following:

2.4.1 Tool 1. Premature Infants' Profile Assessment Sheet: It was including: gender, date of birth, admission date, gestational age/weeks, postnatal-age/days, admission weight/grams, diagnosis, and care unit in which the infant was admitted in.

2.4.2 Tool 2. Infant's Follow-up Assessment Sheet: It was used to collect data about premature infants throughout their stay in the care unit, including: the purpose of needle punctures, date of discharge, C-reactive protein (CRP) weekly profile, and infant's behavioral state (calm, irritable, or mild cry) during needle related invasive procedures.

2.4.3 Tool 3. Infant's Clinical Outcome Assessment Sheet: It was developed to collect data about premature infants at the end of the study period, including: discharge weight/grams, length of hospital stay, average of daily weight gain, culture-proven infection on discharge, number of days on antibiotics, and number of days off antibiotics and infant's outcome on discharge (died, or alive).

2.4.4 Tool 4. Premature Infant Pain Profile (PIPP): It is a standardized scale that was developed 13 years ago and has steadily accumulated the evidence of reliability and validity. [22 \& 23] When compared with other infant pain measures, the PIPP is reported to be one of the most valid and reliable infant acute pain measures available. 
The PIPP consists of 7 indicators, three of them were concerned with behavioral (facial actions: brow bulge, eye squeeze, and naso-labial furrow), 2 physiological (heart rate and oxygen saturation) indicators, and 2 contextual (gestational age and behavioral state) variables that modify pain. For each indicator, the obtained score is ranged between 0 - 3, so in this scale, the total scores were vary from zero to 21 points. Scores equal or lower than 6 indicate that infant has minimal or no pain; scores of $7-12$ indicate slight to moderate pain; and scores above 12 indicate severe pain.

\subsection{Methods}

\subsubsection{Preparatory phase:}

- An Official permission was obtained from the Director of Mansoura University Children Hospital and the head of neonatal care department to conduct the study.

- Ethical approval was obtained from the Research Committee of Faculty of Nursing- Mansoura University.

- The study developed tools were reviewed for their content validity by five expertise in the field of neonatology and necessary modifications were done accordingly.

- The internal consistency of the developed tools was tested for its reliability using alpha Cronpach's test, and it was 0.87 .

- A written informed consent was obtained from the available infant's parent, clarifying their right to withdraw from the research at any time.

- A pilot study was carried out on 7 preterm infants who were admitted in the NCD to evaluate the clarity and applicability of the tools and minor modification was done.

\subsubsection{Field work:}

Researchers, bedside care delivery nurses as well as laboratory technicians performed all study interventions for the admitted premature infants until their discharge. After demographic information was recorded by a bedside nurse in the form of neonatal characteristics at birth, a venous blood sample was collected for bloodstream infection assessment. Topical anesthetic agent (lidocaine 10mg. /dose spray) was applied in 3 applications 2 to 3 minutes before each needle related painful event. Infants were closely monitored for medication side effect (e.g., allergic reactions). Needle punctures to obtain blood samples for culture-proven infection guided by infant's CRP profile were routinely assessed twice/week throughout premature infant's stay in the NCD and at time of discharge. On weekly basis, randomly in association with one needle puncture for assessing CRP, the researchers performed premature infant pain profile (PIPP), and then the mean score was calculated and recorded. PIPP indicators were assessed as the following: (1) score gestational age before infant was examined. (2) Score infant's state before the potentially needle related event by observing the infant for 15 seconds, then record the baseline heart rate and oxygen saturation (physiologic measurements). (3) Assess infant's behavior during needle related event. (4) Observe the infant for 30 seconds immediately following needle related event. Physiologic changes and facial actions/changes seen during this time were scored and recorded immediately. Absent of facial changes was defined as 0 to $9 \%$ of the observation time; minimal, $10 \%$ to $39 \%$ of the time; moderate, $40 \%$ to $69 \%$ of the time; and maximal facial changes as $70 \%$ or more of the observation time.

\subsection{Statistical Analysis:}

The collected data were organized, tabulated and statistically analyzed using SPSS software (Statistical Package for the Social Sciences, version 16, SPSS Inc. Chicago, IL, USA). The normality of data was first tested with one-sample Kolmogorov-Smirnov test. Qualitative data were described using number and percent. Association between categorical variables was tested using Chi-square test $\left(\chi^{2}\right)$. Continuous variables were presented as range, mean \pm standard deviation (SD) for parametric data and Median for non-parametric data. Significance was adopted at $\mathrm{p}<0.05$ for interpretation of results of tests of significance.

\section{Results}

Table (1) revealed that the means, and standard deviations of premature infants' gestational age/week, postnatal-age/days, and admission weight/grams were $32 \pm 2.4,1422 \pm 523$, and $2.6 \pm 4.4$ respectively, $61.1 \%$ of them were girls, $83.3 \%$ of preterm infants diagnosed as prematurity with respiratory distress syndrome, and $80.6 \%$ of them received their care at neonatal intensive care unit.

Table (2) illustrated that all preterm infants were exposed to procedural needle punctures during their stay in the neonatal intensive care unit, with the means, and standard deviations of their exposure times, were $12.4 \pm 5.3$. While $47.2 \%$ of infants were showed quiet awake, opened eyes, no facial movements 15 seconds before needle painful events, $55.6 \%$ of them had calm behavior during needle painful events.

In relation to physiological changes observed 30 seconds after needle painful events; $70.8 \%$, and $94.4 \%$, of the preterm infants had heart rate maximum $0-4 \mathrm{~b} / \mathrm{m}$ increase, and oxygen saturation minimum $0-2.4 \%$ decrease 
respectively. In addition, $65.3 \%, 58.3 \%$, and $73.6 \%$ of them had absent facial actions of frowned forehead, eyes squeezed, and naso-labial furrow respectively. The mean, and standard deviation of total Premature Infant Pain Profile score of the study subjects was $4.5 \pm 2.1$, and $43.1 \%$ of the premature infants had no pain during needle painful events guided by total Premature Infant Pain Profile score.

Table (3) showed that $63.9 \%$ of the preterm infants had no blood-stream infection guided by negative CRP weekly profile, while $12.5 \%$ out of $36.1 \%$ of infants had positive culture once during their staying at the NCD. The means and standard deviations of number of days on, and off antibiotics were $18.8 \pm 10.7$, and 17.5 \pm 7.6 respectively. Regarding to average of daily weight gain and length of stay/days means, and standard deviations were $19.2 \pm 12.0$, and $25.8 \pm 10.9$ respectively.

Table (4) proved that there was no statistically significant relation between C-reactive protein weekly profile in relation to preterm infants' data except for frequency of applying topical anaesthesia and length of stay/ days, at $\mathrm{p}<.001$, and 0.002 respectively.

Vice versa there was statistically significant relation between frequency of applying topical anaesthesia and immunologic parameters presented by average of daily weight gain, number of culture-proven infections and negative C-reactive protein weekly profile, at $\mathrm{p}<.001$, and $\mathrm{p}=.001$ respectively.

Table (5) indicated that there was statistically significant relation between preterm infants' behavioral state during needle painful events and their C-reactive protein weekly profile at $\mathrm{p}<.001$, since the highest number (32) of premature infants who had mixed (almost negative) C-reactive protein weekly profile during their staying at the neonatal care department were behave as calm.

"Table 1": Preterm infants' profile on admission

\begin{tabular}{|lc|}
\hline Item & Subjects $(\mathbf{n}=\mathbf{7 2})$ \\
\hline Gestational age (wk), mean \pm SD wk (range) & $32 \pm 2.4(26-36)$ \\
\hline Admission weight (grams), mean \pm SD (range) & $1422 \pm 523(750-2650)$ \\
\hline Gender & $28(38.9)$ \\
\hline Boy, No. (\%) & $46(61.1)$ \\
\hline Girl, No. (\%) & $2.6 \pm 4.4(0-21)$ \\
\hline & \\
\hline Postnatal-age (days) mean \pm SD (range) & $12(16.7)$ \\
\hline & $60(83.3)$ \\
\hline Diagnosis, No. (\%) & $14(19.4)$ \\
\hline Prematurity and ELBW & $58(80.6)$ \\
\hline Prematurity with RDS & \\
\hline & \\
\hline Care unit & $10(13.9)$ \\
\hline Intermediate & \\
\hline NICU & \\
\hline Bloodstream infection on admission, No. $(\%)$ & \\
\hline Negative & \\
\hline Positive & \\
\hline
\end{tabular}

"Table 2": Needle punctures and its related painful event features among preterm infants

\begin{tabular}{|c|c|}
\hline Item & Subjects $(n=72)$ \\
\hline \multicolumn{2}{|c|}{ Purpose of needle punctures during premature infants' hospital stay, No. (\%) [range]* } \\
\hline Insert long line & $6(8.3)$ [once] \\
\hline Draw capillary blood sample (heel-prick) & $19(26.4)[1-10]$ \\
\hline Draw arterial blood sample & $45(62.5)[1-6]$ \\
\hline Insert peripheral line cannula & $68(94.4)[1-6]$ \\
\hline Draw venous blood sample & $72(100)[2-14]$ \\
\hline Needle exposure times, mean \pm SD (range) & $12.4 \pm 5.3[4-27]$ \\
\hline \multicolumn{2}{|c|}{ Premature infants' states observed 15 seconds before needle painful event, No. (\%) } \\
\hline Quiet sleeping, closed eyes, no facial movements & $2(2.8)$ \\
\hline Active Awake, opened eyes, facial movements present & $14(19.4)$ \\
\hline Active sleep, closed eyes, facial movements present & $22(30.6)$ \\
\hline Quiet Awake, opened eyes, no facial movements & $34(47.2)$ \\
\hline \multicolumn{2}{|c|}{ Premature infants' behaviors during needle painful event, №. (\%) } \\
\hline Calm & $40(55.6)$ \\
\hline Mild Crying & $26(36.1)$ \\
\hline Irritable & $6(8.3)$ \\
\hline
\end{tabular}


"Table 2": Needle Punctures and its related Painful Event Features among Preterm Infants (Cont.)

\begin{tabular}{|c|c|c|}
\hline \multicolumn{2}{|c|}{ Total Premature Infant Pain Profile (PIPP) score, mean \pm SD (range) } & $4.5 \pm 2.1(1-9)$ \\
\hline \multicolumn{3}{|c|}{ Physiological changes and facial actions observed 30 seconds after needle painful event, No. (\%) } \\
\hline \multirow[t]{4}{*}{ Heart rate maximum } & $0-4 \mathrm{~b} / \mathrm{m}$ increase & $51(70.8)$ \\
\hline & $5-14 \mathrm{~b} / \mathrm{m}$ increase & $20(27.7)$ \\
\hline & $15-24 \mathrm{~b} / \mathrm{m}$ increase & $0(0)$ \\
\hline & $\geq 25 \mathrm{~b} / \mathrm{m}$ increase & $1(1.4)$ \\
\hline \multirow[t]{4}{*}{ Oxygen saturation minimum } & $0-2.4 \%$ decrease & $68(94.4)$ \\
\hline & $2.5-4.9 \%$ decrease & $3(4.2)$ \\
\hline & $5-7.4 \%$ decrease & $1(1.4)$ \\
\hline & $7.5 \%$ decrease or more & $0(0)$ \\
\hline \multirow{4}{*}{ Frowned forehead } & Absent & $47(65.3)$ \\
\hline & Minimal & $20(27.8)$ \\
\hline & Moderate & $5(6.9)$ \\
\hline & Maximal & $0(0)$ \\
\hline \multirow[t]{4}{*}{ Eyes squeezed } & Absent & $42(58.3)$ \\
\hline & Minimal & $2(2.8)$ \\
\hline & Moderate & $26(36.1)$ \\
\hline & Maximal & $2(2.8)$ \\
\hline \multirow{4}{*}{ Nasolabial furrow } & Absent & $53(73.6)$ \\
\hline & Minimal & $15(20.8)$ \\
\hline & Moderate & $4(5.6)$ \\
\hline & Maximal & $0(0)$ \\
\hline \multicolumn{3}{|c|}{ Pain intensity during needle painful event guided by total PIPP score, No. (\%) } \\
\hline \multicolumn{3}{|c|}{$\begin{array}{ll}\text { No pain } & 31(43.1) \\
\end{array}$} \\
\hline Minimal pain & & \\
\hline \multicolumn{2}{|l|}{ Mild pain } & \\
\hline
\end{tabular}

* All items are not mutually exclusive

"Table 3": Indicators of preterm infants' bloodstream infection during their staying at the NCD

\begin{tabular}{|lc|}
\hline Item & Subjects (n = 72) \\
\hline Culture-proven infection times guided by CRP weekly profile, No. $\mathbf{\%})$ & \\
\hline Negative & $46(63.9)$ \\
\hline Positive & $26(36.1)$ \\
\hline - Once & $9(12.5)$ \\
\hline - Twice & $5(6.9)$ \\
\hline - Three times & $6(8.3)$ \\
\hline - Four times & $6(8.3)$ \\
\hline & \\
\hline Number of days on antibiotics, mean \pm SD (range) & $18.8 \pm 10.7(3-45)$ \\
\hline Number of days off antibiotics, mean \pm SD (range) & $17.5 \pm 7.6(6-32)$ \\
\hline Average of daily weight gain/g & $19.2 \pm 12.0(20-60)$ \\
\hline Length of stay/ days, mean \pm SD (range) & $25.8 \pm 10.9(5-57)$ \\
\hline
\end{tabular}

"Table 4": Statistical relations between CRP weekly profile and preterm infants' data and frequency of applying topical anaesthesia and immunologic parameters

\begin{tabular}{|lc|}
\hline Item & Subjects $(\mathbf{n}=\mathbf{7 2})$ \\
\hline CRP weekly profile in relation to premature infants' data $\boldsymbol{\chi}^{\mathbf{2}}$ (p-value) & \\
\hline Gestational age & $95.2(.12)$ \\
\hline Postnatal age on admission & $79.25(.89)$ \\
\hline Admission weight & $508.4(.08)$ \\
\hline Diagnosis & $57.6(.67)$ \\
\hline Number of days on antibiotics & $279.4(.25)$ \\
\hline Frequency of applying topical anaesthesia & $230.1(<.001)^{*}$ \\
\hline Purpose of needle punctures & $63.4(.02)$ \\
\hline CRP admission results & $29.3(.12)$ \\
\hline CRP discharge results & $16.8(.02)$ \\
\hline Length of stay/ days & $289.2(.002)^{*}$ \\
\hline & \\
\hline Frequency of applying topical anaesthesia in relation to immunologic \\
\hline parameters, $\boldsymbol{\chi}^{\mathbf{2}}$ (p-value) & $274.5(<.001)^{*}$ \\
\hline Average of daily weight gain & $130.6(<.001)^{*}$ \\
\hline Number of culture-proven infections & $111.0(.001)^{*}$ \\
\hline Negative CRP weekly profile & $659.6(.07)$ \\
\hline Length of stay/ days & \\
\hline *Significant $(\mathbf{P}<\mathbf{0 . 0 5 )}$ & \\
\hline
\end{tabular}


The Effect of Numbing Needles Pain with Topical Anaesthesia on Preterms' Exposure to Neonatal

"Table 5": Statistical relation between preterm infants' behavioral state during needle painful events and their CRP weekly profile

\begin{tabular}{|c|c|c|c|c|c|c|c|}
\hline \multicolumn{3}{|l|}{ Item } & \multicolumn{5}{|c|}{ Subjects $(n=72)$} \\
\hline & \multicolumn{7}{|c|}{ State } \\
\hline & & Calm & Irritable & $\begin{array}{c}\text { Mild } \\
\text { Crying }\end{array}$ & Total & $\chi^{2}$ & p-value \\
\hline \multirow{4}{*}{$\begin{array}{l}\text { CRP } \\
\text { weekly } \\
\text { profile }\end{array}$} & Negative & 6 & 0 & 6 & 12 & \multirow[t]{4}{*}{47.69} & \multirow[t]{4}{*}{$<.001 *$} \\
\hline & Mixed (almost negative) & 32 & 5 & 6 & 43 & & \\
\hline & Posit ve & 0 & 0 & 5 & 5 & & \\
\hline & Mixed (almost positive) & 2 & 1 & 9 & 12 & & \\
\hline \multicolumn{2}{|l|}{ Total } & 40 & 6 & 26 & 72 & & \\
\hline
\end{tabular}

\section{Discussion}

Neonatal sepsis remains a feared cause of morbidity and mortality in the neonatal period, both among term and preterm infants. [24] Maternal, neonatal, and environmental factors are associated with risk of infection, leading to 2.9 million global neonatal deaths. [25 \& 26] Strong evidence supports acute pain as a stressor that impairs immune function. Given the vital link between immunity and the defense against bacterial and viral infections, it is incumbent upon nurses to preserve immune capacity to prevent neonatal infection. [7]

A study conducted by Donia and Tolba [41] showed that sick and preterm infants are exposed to repeated procedural pain-related stress, as part of their care in the Egyptian neonatal intensive care unit per day, with the frequency of exposure increased during the first few days of their life. [27] In his study, Noel [28] added that children can remember painful experiences, and this can affect how they experience future painful episodes in their childhood and may extend even to their adult lives. A review by the World Health Organization (WHO) has identified pain in the pediatric setting as an important public health problem. [29] It is therefore imperative that neonatal pain be anticipated and managed appropriately. Commonly encountered pain in the NICUs can be categorized as pain associated with infection.[30] The present study revealed that the means, and standard deviations of premature infants' gestational age/week, postnatal-age/days, and admission weight/grams were $32 \pm 2.4,1422 \pm 523$, and $2.6 \pm 4.4$ respectively. In this context, Edwards [31] reported that neonatal sepsis is a systemic infection occurring in infants at $\leq 28$ days of life, and Benitz [32] illustrated that, infant factors associated with early-onset sepsis include prematurity/low birth weight.

The present study showed that the majority of premature infants had positive bloodstream infection on admission, this was agreed with the subsequent WHO Young Infants Clinical Signs Study (YICSS), focused on clinical signs detected by primary care health workers for 3177 neonates in the first week of life attending health-care facilities in countries within sub-Saharan Africa, south Asia, and Latin America. The presence of any one of seven clinical signs and symptoms predicted severe bacterial illness (on the basis of an experienced pediatrician's assessment) with a sensitivity of $85 \%$ and specificity of $75 \%$, in those seeking care. [33] During hospital stay all premature infants in the present study were exposed to procedural needle punctures and more than half and less than half of them behave calmly and had minimal pain intensity during it respectively as well. Yuhico [30] found that needle punctures are one of the most common procedures that children experience as part of routine medical care. Needle procedures include injections (intramuscular or subcutaneous), venipuncture and venous cannulations. In the same line a prospective cross-sectional survey carried out in US children's hospital reported that worst pain was $40 \%$ cause of needle poke. [34]

According to Witt [35], EMLA (a mixture of lidocaine $2.5 \%$ and prilocaine $2.5 \%$ ) has been well established as effective in the neonatal population for reducing pain associated with minor procedures, such as circumcision, venipuncture, lumbar punctures, and during peripherally inserted central catheter (PICC) line placement. Similar to the study findings, Taddio [36] illustrated that, when performing venipuncture, it is recommended that clinicians use topical analgesic, as it reduces pain. Further to Kaur [42] stated that EMLA or its composition should also be used for analgesia with lumbar punctures, as it reduced heart rate variability, facial grimacing, and oxygen desaturations when compared to placebo. Yuhico [30] explained that the use of topical local anaesthetics provide analgesia by blocking nociceptive transmission in nerve cells. He added that application may be noninvasive (topical anesthetic), which considered safe for children for all ages. [37]

The current study showed that, there was statistically significant relation between frequency of applying topical anaesthesia and immunologic parameters presented by average of daily weight gain, number of culture-proven infections, and negative CRP weekly profile. This was constant with Page [38] who explore that immune function is another area impacted by early experiences with pain or stress and consistently shown in animal studies. The current study indicated that there was statistically significant relation between premature infants' behavioral state during needle painful events and their CRP weekly profile. Anand [39] revealed that painful events can affect complex behavioral responses for up to $24 \mathrm{~h}$ after the event; altered sleep-wake cycles, feeding, and crying have been documented in male infants circumcised without local anesthetic. Therefore, in neonates, topical anesthesia reduce behavioral pain response to vein-puncture but not heel lance. [40] 


\section{Conclusion And Recommendations}

In summary, premature infants are vulnerable to infection associated with repeated exposure to needle related painful procedures when admitted to the NCD. It can be concluded that, topical anesthetic/or analgesic regimen can influence immunomodulation or boost immune function significantly to avert sepsis; presented by significant improvement among immunologic parameters in the form of average of daily weight gain, number of culture-proven infections, and negative CRP weekly profile. Moreover, numbing procedural pain through the use of topical local anesthetic can provided control over the behavioral state of preterm infants.

\section{Limitation of the study}

Interpretations of the study results should acknowledge a limitation of we did not include a control group that would have allowed us to determine the absolute efficacy of analgesic intervention. However, we felt that it would be unethical to prohibited preterm infants from analgesia for the purposes of our study.

Based on the results of the present study, the following recommendations are suggested:

1- Topical anaesthesia should be utilized for procedural pain management in the NICUs to reduce the adverse effect of pain on preterm infants' immunity and its subsequent outcomes.

2- Recognition of neonatal pain as a valid concern then protocolize stepwise treatment plan for the procedures encountered in the NICU following the tiered approach to neonatal pain management.

3- Continued auditing to ascertain appropriate treatment for neonatal pain.

\section{Author Disclosure Statement}

No competing financial interests and/or associations that pose real or perceived conflicts of interest in connection with the article exist.

\section{References}

[1]. Fitzgerald M, Walker SM. Infant pain management: a developmental neurobiological approach. Nat Clin Pract Neurol. 2009; 5: 3550. [PubMed: 19129789]

[2]. Carbajal R, Rousset A, Danan C, et al. Epidemiology and treatment of painful procedures in neonates in intensive care units. JAMA. 2008;300:60-70.

[3]. Li J, Walker SM, Fitzgerald M, Baccei ML. Activity-dependent modulation of glutamatergic signaling in the developing rat dorsal horn by early tissue injury. J Neurophysiol. 2009; 102: 2208-2219. [PubMed: 19675290]

[4]. O'Sullivan AT, Rowley S, Ellis S, Faasse K, Petrie K. The Validity and Clinical Utility of the COVERS Scale and Pain Assessment Tool for Assessing Pain in Neonates Admitted to an Intensive Care Unit. Clinical Journal of Pain. 2016; 32: 51-57.

[5]. Czarnecki ML, Turner HN, Collins PM, et al. Procedural pain management: a position statement with clinical practice recommendation. Pain Manag Nurs. 2011; 12(2): 95-101.

[6]. Nimbalkar A, Dongara AR, Phatak AG, Nimbalkar SM. Knowledge and attitudes regarding neonatal pain among nursing staff of pediatric department: an Indian experience. Pain Management Nursing 2012 Available at www.sciencedirect.com/science/ article/pii/S1524904212000847.

[7]. Hatfield LA. Does mitigating pain decrease the risk of infection? The interactions between nociception and immune function. J. of Neonatal Nursing. 2015; 21: 11-17

[8]. Calvo M, Dawes JM, Bennett DL. The role of the immune system in the generation of neuropathic pain. Lancet Neurol. 2012; 11(7): 629-642.

[9]. Grace PM, Hutchinson MR, Maier SF, Watkins LR. Pathological pain and the neuroimmune interface. Nature reviews. Immunology. 2014; 14(4): 217-231.

[10]. Ren K, Dubner R. Interactions between the immune and nervous systems in pain. Nat. Med. 2010; 16(11): 1267-1276.

[11]. Stein C, Machelska H. Modulation of peripheral sensory neurons by the immune system: implications for pain therapy. Pharmacol. Rev. 2011; 63(4): 860-881.

[12]. Chiu IM, Heesters BA, Ghasemlou N, Von Hehn CA, Zhao F, Tran J, Woolf CJ. Bacteria activate sensory neurons that modulate pain and inflammation. Nature. 2013; 501(7465): 52-57.

[13]. Hatfield LA, Umberger R. Does mitigating pain decrease the risk of infection? The interactions between nociception and immune function. Journal of Neonatal Nursing. 2015; 21: 11-17.

[14]. Vinall J, Grunau RE. Impact of repeated procedural pain-related stress in infants born very premature. Pediatr Res. 2014; 75(5): 584-587.

[15]. van Dijk M, Tibboel D. Update on pain assessment in sick neonates and infants. Pediatr Clin North Am. $2012 ; 59: 1167-1181$. [PubMed: 23036250]

[16]. Johnston C, Barrington KJ, Taddio A, Carbajal R, Filion F. Pain in Canadian NICUs: have we improved over the past 12 years? Clin J Pain. 2011; 27: 225-232. [PubMed: 21178602]

[17]. Tadio A, Lee C, Yip A, Parvez B, McNamara PJ, Shah V. Intravenous morphine and topical tetracaine for treatment of pain in premature neonates undergoing central line placement. JAMA 2006; 295: 793-800.

[18]. Berkman S, MacGregor J, Alster T. Adverse effects of topical anesthetics for dermatologic procedures. Informa Healthcare. 2012; 11(3): 415-423.

[19]. O'Brien L, Taddio A, Lyszkiewicz DA, Koren G. A critical review of the topical local anesthetic amethocaine (Ametop) for pediatric pain. Paediatr Drugs. 2014; 7: 41-54.

[20]. Hall RW, Anand, K. Pain Management in Newborns. Clinics in Perinatology. 2014; 41(4): 895-924.

[21]. Schack-Nielsen L, Michaelsen KF. Advances in our understanding of the biology of human milk and its effects on the offspring. J Nutr. 2007; 137(2): 503-510

[22]. Ballantyne M, Stevens B, McAllister M, et al. Validation of the premature infant pain profile in the clinical setting. Clin J Pain. 1999; 15: 297-303. 
[23]. McNair C, Ballantyne M, Dionne K, et al. Postoperative pain assessment in the neonatal intensive care unit. Arch Dis Child Fetal Neonatal. 2004; 89: 537-541.

[24]. Camacho-Gonzalez A, Spearman PW, \& Stoll BJ. Neonatal infectious diseases: evaluation of neonatal sepsis. Pediatr Clin North Am. 2013; 60(2): 367-89. doi: 10.1016/j.pcl.2012.12.003.[PMC free article] [PubMed] [Cross Ref]

[25]. Liu L, Johnson HL, Cousens S. Global, regional, and national causes of child mortality: an updated systematic analysis for 2010 with time trends since 2000. Lancet. 2012; 379: 2151-2161.[PubMed]

[26]. UNICEF Committing to child survival: a promise renewed. Progress report 2013.http:/ /www. unicef.org/publications/ files/APR_Progress_Report_2013_9_Sept_2013.pdf (accessed Dec 4, 2013).

[27]. Simons SH, van Dijk M, Anand KS, Roofthooft, D, van Lingen RA, \& Tibboel D. Do we still hurt newborn babies? A prospective study of procedural pain and analgesia in neonates. Arch. Pediatr. Adolesc. Med. 2003; 157: 1058-1064

[28]. Noel M, McMurtry CM, Chambers CT, \& McGrath PJ. Children's memory for painful procedures: the relationship of pain intensity, anxiety, and adult behaviors to subsequent recall. J Pediatr Psycho.1 2010; 35: 626-636.

[29]. World Health Organization. WHO guidelines on the pharmacological treatment of persisting pain in children with medical illnes ses. WHO guidelines Approved by the Guidelines Review Committee. Geneva: WHO; 2012. Available online at: http://whqlibdoc.who.int/publications/2012/9789241548120_Guidelines.pdf (accessed September 2014.

[30]. Yuhico AM, \& Mb Bb JC. Identifying and managing pain appropriately in children is a challenging but vital role for GPs. Both pharmacological and non-pharmacological interventions can help relieve and decrease pediatric pain. Medicine Today. 2014; 15(10): 26-32.

[31]. Edwards MS, \& Baker CJ. Sepsis in the newborn. 2004; 545-561In Gershon AA, Hotez PJ, \& Katz SL, editors. (ed), Krugman's infectious diseases of children. 11th ed. Mosby, Philadelphia, PA.

[32]. Benitz WE, Gould JB, \& Druzin ML. Risk factors for early-onset group B streptococcal sepsis: estimation of odds ratios by critical literature review. Pediatrics1999; 103: e77. 10.1542/peds.103.6.e77 [PubMed] [Cross Ref].

[33]. Seale AC, Blencowe H, Manu AA, Nair H, Bahl R, Qazi SA, Zaidi AK, Berkley JA, Cousens SN, Lawn JE, \& for the pSBI Investigator Group. Estimates of possible severe bacterial infection in neonates in sub-Saharan Africa, south Asia, and Latin America for 2012: a systematic review and meta-analysis. Lancet Infect Dis. 2014; 14(8): 731-741.

[34]. Friedrichsdorf SJ. Postier AC, Eull D, Foster L, Veidner C, \& Compbell F. Hospital Pediatrics. 2015; 5(1): 18-26.

[35]. Witt N, Coynor S, Edwards C, Bradshaw H. A Guide to Pain Assessment and Management in the Neonate. Current Emergency and Hospital Medicine Reports. 2016; 4(1): 1-10

[36]. Taddio A, Ohlsson A, Einarson TR, et al. A systematic review of lidocaine-prilocaine cream (EMLA) in the treatment of acute pain in neonates. Pediatrics. 1998;101:E1CrossRefPubMed

[37]. www.hc-sc.gc.ca/dhp-mps/medeff/advisories-avis/puplic/_2009/emla ametop_pc-cp-eng.php

[38]. Page GG. Are there long-term consequences of pain in newborn or very young infants? 2004; 13(3): 10-17.

[39]. Anand KJ, \& Hickey PR. Pain and its effects in the human neonate and fetus. N. Engl. J. Med. 1987; 317: 1321-1329.

[40]. Attarian S, Tran LC, Aimee Moore A, Stanton G, Meyer E, \& Moore RP. The neuro developmental impact of neonatal morphine administration Brain Sci. 2014; 4: 321-334. doi:10.3390/brainsci4020321.

[41]. Donia AE, \& Tolba OA. Effect of early procedural pain experience on subsequent pain responses among premature infants. Egyptian Pediatric Association Gazette. 2016; 64: 74-80. doi.org/10.1016/j.epag.2016.03.002

[42]. Kaur G, Gupta P, Kumar A. A randomized trial of eutectic mixture of local anesthetics during lumbar puncture in newborns. Arch Pediatr Adolesc Med. 2003; 157: 1065 